\title{
Does Gel Affect Cytology or Comfort in the Screening Papanicolaou Smear?
}

\author{
Meredith Gilson, Maj, USAF, MC, Alpa Desai, Capt, USAF, MC, \\ Gabriella Cardoza-Favarato, Maj, USAF, MC, Penny Vroman, Capt, USAF, MC, and \\ Jennifer A. Thornton, PhD
}

Purpose: Current medical training recommends obtaining cervical cytological specimens without the use of lubricating gel. The purpose of this study was to determine whether water-soluble lubricant gel affects cytologic outcomes in the screening Papanicolaou smear and patient comfort during vaginal speculum examination.

Methods: The study was a randomized controlled trial performed at David Grant US Air Force Medical Center (Travis Air Force Base, CA). Participants were female patients at least 18 years old presenting for an annual Papanicolaou smear. Each patient, blinded to group assignment, consented to two consecutive Papanicolaou smears. The first Papanicolaou smear was performed without gel in all subjects as part of the "standard of care." Thirty control patients underwent a second examination with no gel, and 40 other patients had the second examination with gel. All patients rated the discomfort of each Papanicolaou smear on a numerical pain scale. Main outcome measures were cytologic discrepancies on standard glass slide samples and comfort differences regarding the use of gel lubrication. Fisher's exact test was used to interpret the effect of gel on cytology results. Student's $t$ test was performed to compare the discomfort ratings for the second Papanicolaou smear in the GEL vs. the NO GEL groups.

Results: There was no statistically significant difference between the number of inadequate Papanicolaou smears $(P=.50)$ nor in the discomfort level ratings in the GEL vs. the NO GEL groups $(P=.69)$.

Conclusion: Speculum gel lubrication does not affect cervical cytology during the traditional Papanicolaou smear, nor does it provide significant alteration of patient discomfort. (J Am Board Fam Med 2006;19:340-4.)

The Papanicolaou smear is an effective and wellaccepted screening examination for the early detection of cervical cellular abnormalities. It has significantly decreased the morbidity and mortality from cervical cancer and is now a standard part of preventive health care for women of reproductive age. $^{1,2}$ It is therefore important to get the most accurate and reliable cell specimens by using proper techniques.

Despite its clinical importance, the Papanicolaou smear examination can be an awkward and

Submitted 2 May 2005; revised 13 December 2005; accepted 22 December 2005.

From American Academy of Family Physicians, Uniformed Services Academy of Family Practice, Hanscom Air Force Base, MA.

Funding: The work reported herein was performed and funded under United States Air Force Surgeon Generalapproved Clinical Investigation No. 20020003H. Other sources of support include Family Practice Clinic, David Grant Medical Center (Travis Air Force Base, CA).

Conflict of interest: none declared.

Corresponding author: Dr. Meredith Gilson, Maj, USAF, MC, MD, 5 Shaw Circle, Hanscom AFB, MA 01731 (Email: meredith.gilson@hanscom.af.mil). unpleasant experience for many women. Physicians should thus be sensitive to this issue and take measures to make the smear as comfortable as possible for the patient.

In medical training, students/residents are taught never to use water-soluble gel to lubricate the speculum in the collection process. ${ }^{4,5}$ The presumable theory behind this teaching is that the gel may obscure the cellular preparation via gel overlay or altered uptake of dye in staining. However, a lack of literature exists to support this dogma, and recent literature refutes it. ${ }^{6,7}$ Discussion with colleagues shows that many feel the use of gel lubricant allows for easier entry of the speculum into the vagina and that it is more comfortable for the patient.

The goals of the current study were to formally investigate whether gel obscures cervical cytology and whether it decreases pain and discomfort for patients. To this end, two Papanicolaou smears were performed on all subjects: the first was performed without cervical gel (to ensure that patients would not require a second visit in case the gel 
obscured the cervical cytology), and the second used cervical gel for half the patients and no cervical gel for the other half of the patients. Our design thus used each patient as her own control, randomized the use of gel or no gel between patients, and ensured that cytotechnologists and patients were blinded to the method used.

\section{Methods}

The study was conducted at David Grant US Air Force Medical Center Family Practice Clinic (Travis Air Force Base, CA) between July 2001 and May 2002. Female patients at least 18 years of age who presented for a routine annual gynecologic examination were screened for participation. All eligible patients were given the opportunity to participate in the study. Patients were excluded if they did not have a cervix, had an infectious gynecologic complaint, conveyed an extreme aversion to Papanicolaou smears (as assessed by the provider), or had chronic pelvic pain.

Informed consent was obtained from eligible and willing patients. Information documenting parity, history of abnormal Papanicolaou smears and cervical procedures, current menopausal status, current hormone replacement use, current oral contraceptive use, and current intrauterine device use were documented on a patient data sheet. All patients then underwent two consecutive Papanicolaou smears using plastic speculums. In all patients, the first Papanicolaou smear was performed using a dry speculum, with no water or gel as per the standard of care. The second Papanicolaou smear was performed using a 2.7 -g packet of waterbased lubricant gel applied to the external surfaces of the blades of the speculum in approximately half the patients (GEL group) and without gel or water in the rest of the patients (NO GEL group); a computer-generated random number table assigned patients to the GEL vs. the NO GEL group. The patients were blinded as to which group they were randomized. All Papanicolaou smears were performed by one of five physicians in the Family Practice clinic at the level of second year resident or above.

An appropriately sized Welch-Allyn plastic bivalve speculum was chosen by the physician based on the anatomy of the patient. The majority of Papanicolaou smears were done with medium-sized (green) speculums. After insertion of the speculum for both smears, the physician used a large cotton- tipped swab as needed for clearing of cervical mucous. Ectocervical cells were collected with a wooden spatula and endocervical cells were collected with a cytobrush. Cells from all Papanicolaou smears were applied to glass slides and sprayed with fixative. All slides were then forwarded to cytotechnologists who were blinded to the group assignments. Samples were recorded as "unsatisfactory" if no endocervical cells were present, if they were obscured by blood, or if drying artifact or gel overlay were present; otherwise, they were recorded as "satisfactory."

In addition, after each Papanicolaou smear each patient was asked to rate her discomfort on the Wong-Baker Faces Pain Rating Scale ${ }^{3}$ with numbers ranging from 0 (no discomfort) to 10 (most discomfort).

\section{Data Analysis}

SPSS version 12.0 for Windows was used for all analyses; the $\alpha$ level was set at $0.05 . \chi^{2}$ tests of independence and Fisher's exact tests (when assumptions regarding minimum expected cell counts were violated) were used for categorical level data analyses. Because the sample size was relatively large, we used a Student's $t$ test rather than the Mann-Whitney $U$ test to analyze the discomfort level data; in general, the central limit theorem holds for $\mathrm{n}>30 .{ }^{8}$ (Note: statistical analyses performed using the Mann-Whitney $U$ test yielded identical conclusions.)

\section{Results}

\section{Sample Characteristics}

Seventy patients participated in this study. Twelve additional patients met criteria, but declined participation. Because the assignment to groups was random, the final group sizes were unequal; 30 patients were randomly assigned to the NO GEL group and 40 patients were randomly assigned to the GEL group.

Demographic characteristics of each group are shown in Table 1. Parity is expressed as the number of subjects having previously delivered children; abnormal Papanicolaou smear/cervical procedure history is expressed as the number of subjects having one or more abnormal Papanicolaou smears or other cervical procedure in their lifetime; postmenopausal is expressed as the number of subjects who have entered menopause. No significant rela- 


\begin{tabular}{|c|c|c|c|c|c|}
\hline \multirow[b]{2}{*}{ Measure } & \multicolumn{2}{|c|}{$\begin{array}{l}\text { GEL Group } \\
(\mathrm{N}=40)\end{array}$} & \multicolumn{2}{|c|}{$\begin{array}{l}\text { NO GEL Group } \\
(\mathrm{N}=30)\end{array}$} & \multirow[b]{2}{*}{$P$ Value } \\
\hline & $\mathrm{N}$ & $\%$ & $\mathrm{~N}$ & $\%$ & \\
\hline Parity & 31 & 78 & 24 & 80 & .80 \\
\hline Abnormal Papanicolaou smear/cervical procedure history & 14 & 35 & 6 & 20 & .17 \\
\hline Postmenopausal & 5 & 13 & 5 & 17 & .74 \\
\hline Current hormone replacement therapy use & 1 & 3 & 2 & 7 & .57 \\
\hline Current oral contraceptive use & 10 & 25 & 9 & 30 & .64 \\
\hline Current intrauterine device use & 0 & 0 & 1 & 3 & .43 \\
\hline
\end{tabular}

tionship was found between group assignment (GEL vs. NO GEL) and any of the demographic characteristics.

\section{Number of Unsatisfactory Samples}

Slides taken from the second smear were used to answer our research question regarding whether using gel results in a higher proportion of unsatisfactory samples, since the first set of samples were all taken without gel for clinical purposes (note: from the first set, only 2 samples were deemed unsatisfactory: 1 was obscured by blood, and 1 had poor fixation). Due to technician error and improper slide preparation, 4 slides from the second smears ( 3 in the GEL group and 1 in the NO GEL group) had no cytopathology performed; the number of unsatisfactory slides among the remaining 66 was compared between groups (see Table 2).

A Fisher's exact test showed no statistically significant difference between the proportion of unsatisfactory Papanicolaou smears in the GEL vs. the NO GEL groups $(P=.50)$. The only two unsatisfactory slides had obscuring blood. Thus, no slides were deemed unsatisfactory because of cervical gel.

\section{Discomfort Ratings}

A Student's $t$ test comparing the discomfort ratings for the second Papanicolaou smear in the GEL

Table 2. Cytopathologic Results

\begin{tabular}{lrrrrr}
\hline & \multicolumn{2}{c}{ GEL Group } & & \multicolumn{2}{c}{ NO GEL } \\
\cline { 2 - 3 } \cline { 5 - 6 } Slide Quality & $\mathrm{N}$ & $\%$ & & $\mathrm{~N}$ & $\%$ \\
\hline Unsatisfactory & 2 & 5 & & 0 & 0 \\
Satisfactory & 35 & 87.5 & & 29 & 97 \\
Not performed & 3 & 7.5 & & 1 & 3 \\
\hline
\end{tabular}

$($ mean $=1.8 ; \mathrm{SD}=1.4)$ vs. the NO GEL $($ mean $=$ 1.7 ; $\mathrm{SD}=1.3$ ) group showed no statistically significant difference $(P=.69$; see Figure 1$)$. In addition, we looked at "change" in discomfort level ratings by subtracting the score from the second Papanicolaou smear from the first Papanicolaou smear in each subject and comparing the NO GEL group to the GEL group. Thus, a negative change score indicates worsening comfort level, and a positive change score indicates improvement in comfort level. This method allowed us to control for baseline discomfort level scores from the first $\mathrm{Pa}$ panicolaou smears, which were performed without gel in each group. (Note: the discomfort level ratings from the first set of Papanicolaou smears were not significantly different between the two groups; mean difference $=0.3 ; P=.45$.). An analysis of the change scores supported our findings $(P=.57)$ of no difference in discomfort ratings between the GEL (mean change $=0.5 ; \mathrm{SD}=1.5$ ) and the $\mathrm{NO}$ GEL group (mean change $=0.3 ; \mathrm{SD}=1.5$ ), although both groups showed slightly less discomfort during the second Papanicolaou smear.

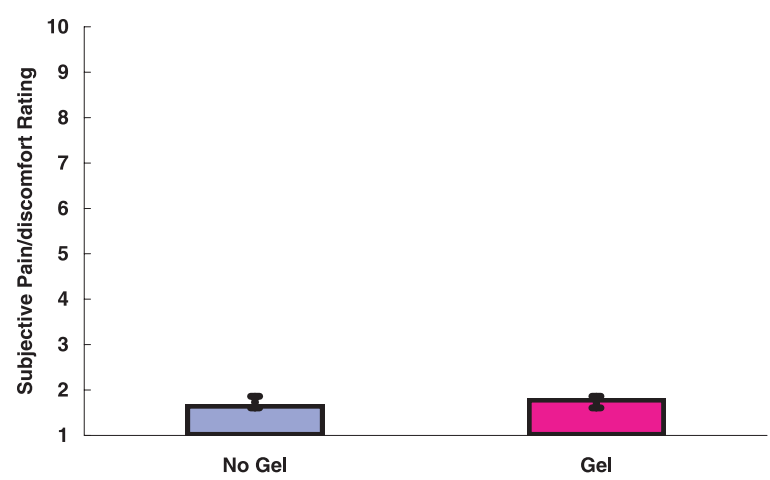

Figure 1. Comparison of discomfort level of second Papanicolaou smear; error bars represent the SD in each group. 


\section{Power/Sample Size Calculations}

Because no range or standard deviation estimates (necessary to calculate the effect size) were available before the study, a priori sample size/power calculations were not performed. However, post hoc power analyses using the obtained standard deviations were performed using nQuery Advisor, version 5.0 (two-tailed significance level $=0.05$; desired power $\geq 80 \%$ ). Our Fisher's exact test comparing the proportion of unsatisfactory samples had $83 \%$ power to detect a difference between a NO GEL group proportion of 0.03 (3\%) and a GEL group proportion of 0.33 (33\%) with a sample size of at least 30 in each group. Thus, our study was powerful enough to detect a 10 -fold difference in the number of unsatisfactory slides between the two groups. Our Student's $t$ test looking at the subjective pain/discomfort survey results had $83 \%$ power to detect a 1-unit difference in mean discomfort level assuming a SD of 1.4 with a sample size of at least 30 in each group.

\section{Discussion}

Our study showed that there was no difference in the proportion of unsatisfactory samples when gel was used for collection of Papanicolaou smears. However, no difference in discomfort level was found in women who received gel lubrication relative to those who did not receive gel lubrication, and the change in discomfort level from the first to the second Papanicolaou smear also did not differ between the groups.

Our results are interesting in light of classic teaching. For years, medical standard of care discouraged the use of speculum lubrication during the gynecologic examination for fear of contaminating pathology results. Current, commonly referenced textbooks cite the following methods for performing the Papanicolaou smear: "The speculum is placed in the vagina after being lubricated with water only. . , 4 " and, "Insertion of the unlubricated speculum should precede the digital examination of the vagina because the presence of lubricating jelly on the cellular specimens interferes with the Papanicolaou stain." Our study contradicts these textbooks' assertions and instead supports the hypothesis that the use of water-soluble gel lubrication on the plastic vaginal speculum does not significantly interfere with interpretation of cytological results. In the textbook, Procedures for
Primary Care Physicians, Pfenninger does advise the use of a "small amount of water-soluble lubricant on the warmed speculum" before inserting. ${ }^{9}$

Our data contribute to the recently expanding literature regarding the use of speculum lubrication during the Papanicolaou smear and its effect on cervical cytology results. ${ }^{6,7}$ In one study, a watersoluble lubricant was applied at the vaginal introitus via a gloved finger before the insertion of an externally lubricated speculum, ${ }^{7}$ whereas in the other, water-soluble gel lubricant was used on the outer inferior blade of the plastic speculum. ${ }^{6}$ Our study used water-soluble gel lubricant applied to the outer superior and inferior blades of the plastic speculum. In both previous studies, there was no statistically significant difference in cervical cytology results when using gel lubrication. Likewise, the data from our study reveal no unsatisfactory cytopathologic results due to gel overlay.

Although our study used fewer patients than previous studies, unlike other studies, we examined results on the same patients with and without gel, and post hoc power analyses showed that our sample size was sufficient to detect large differences between groups in the number of unsatisfactory slides and small differences (ie, 1 unit) in discomfort level. Also, unlike other studies, we measured patient pain/discomfort levels when using gel lubrication, but found no statistically significant difference in discomfort level between women who received gel lubrication and those who used no lubrication. Our discomfort level ratings were relatively low in both groups (ie, there was a floor effect, making it difficult to detect any difference between groups), possibly because our clinic population contains primarily premenopausal females who generally do not have problems with vaginal dryness. In postmenopausal patients, vaginal dryness and atrophic vaginal epithelium can contribute to the discomfort of the Papanicolaou smear examination; future studies limited to this population may show a more beneficial effect of gel lubrication on reducing discomfort. The proficiency level of the providers performing the examination may have also contributed to the low overall discomfort ratings. The providers had numerous years of experience in performing examinations and were thus more skilled than a physician beginning his/her training. Finally, it is worth noting that the numerically lower (but nonsignificant) discomfort level after the second Papanicolaou smear in each group 
could have been due to a relaxation of the introital muscles. A different approach to the discomfort factor could have been to ask women which Papanicolaou smear they preferred; this may be interesting to explore in future studies.

Although we inserted the speculum twice, this should not have affected our conclusions because both groups had an initial Papanicolaou smear performed without gel and had their discomfort level evaluated twice. This allowed us to look at change in discomfort levels within groups as well as discomfort level differences between groups. If the second insertion was more uncomfortable (because of trauma or tears) or more comfortable (because of relaxation of introital muscles), then it should have been consistently so in each group.

Our results support the idea that gel lubrication does not affect the results of the Papanicolaou smear, but they also show no significant alteration of pain/discomfort level. We therefore advise that the use of speculum gel lubrication in the female Papanicolaou smear be regarded as an option for physicians based on patient preference. In particular, for patients with difficult to locate cervices, providers can feel more comfortable performing the bimanual examination with gel lubrication without concern for interfering with cell collection.

\section{Limitations}

One flaw in our study was that the providers were not blinded as to the randomization group of the patient. Although the patient and the pathologists were kept blinded, the provider was aware whether they were using gel during the examination. However, we believe that this was unlikely to have affected our results. Also, our demographic population consisted primarily of low-risk patients and excluded any patients with infectious complaints or chronic pelvic pain. Future studies may include the effect of speculum gel lubrication on the diagnosis of female vaginal infections, as well as the applicability of these results when dealing with a high-risk patient population or chronic pain patients. Our study had a relatively low sample size, and although power analyses showed that this was sufficient to detect small differences in discomfort level between the groups, they also showed that that it was only sufficient to detect relatively large differences regarding whether the slides were satisfactory. Future studies with larger sample sizes would thus be informative. Approximately $80 \%$ of our study popu- lation was parous women, who due to prior childbirth may have less discomfort associated with the Papanicolaou examination. Further research could include a larger number of nulliparous women to better assess effect of parity on discomfort with gel versus no gel. It would also be interesting to address whether including women with extreme aversion to Papanicolaou smears would change the comfort level results and also to collect data concerning speculum size versus anatomic size. Finally, traditional Papanicolaou smears have largely been replaced by the Thin Prep technique, which provides another opportunity for future research on this topic.

\section{Summary}

The use of water-soluble lubricant gel in the collection of the traditional Papanicolaou smear did not interfere with the cervical cytology results nor did it make a difference in the discomfort level of the patients in our sample.

\section{References}

1. Stenkvist B, Bergstrom R, Eklund G, Fox CH. Papanicolaou smear screening and cervical cancer: what can you expect? JAMA 1984;252:1423-6.

2. Day NE. Effect of cervical cancer screening in Scandinavia. Obstet Gynecol 1984;63:714-8.

3. Wong DL, Hockenberry-Eaton M, Wilson D, Winkelstein ML, Schwartz P. Wong's essentials of pediatric nursing. 6th ed, St. Louis: Mosby; 2001. p. 1301.

4. DeCherney A, Nathan L. Current obstetric and gynecologic diagnosis and treatment, The McGrawHill Companies: 2003. Available from: http://www. online.statref.com/document.aspx? fxid $=30 \&$ docid $=351$ [26 May 2003].

5. Degowin R. Degowin's Diagnostic Examination, The McGraw-Hill Companies, 2000. Available from: http://www.online.statref.com/document. aspx? fxid=36\&docid=242 [26 May 2003].

6. Amies AM, Miller L, Lee SK, Koutsky L. The effect of vaginal speculum lubrication on the rate of unsatisfactory cervical cytology diagnosis. Obstet Gynecol 2002;100(5 Pt 1):889-92.

7. Harer WB, Valenzuela G Jr., Lebo D. Lubrication of the vaginal introitus and speculum does not affect the Papanicolaou smear. Obstet Gynecol 2002;100(5 Pt 1):887-8.

8. Ott L. An introduction to statistical methods and data analysis. 3rd ed. Boston (MA): PWS-Kent Publishing Company; 1988. p. 113.

9. Pfenninger JL, Fowler GC. Procedures for primary care physicians. 2 ed. St. Louis (MO): Mosby, Inc.; 2003. p. 1153. 